SUBJECT AREAS:

OVARIAN CANCER

TRANSLATIONAL RESEARCH

Received

21 August 2013

Accepted

24 March 2014

Published

9 May 2014

Correspondence and requests for materials should be addressed to

A.D. (anna.defazio@ sydney.edu.au)

* A full list of authors for the Australian Ovarian

Cancer Study Group and their affiliations appears at the end of the paper.

$\dagger$ These authors contributed equally to this work.

\section{Paclitaxel sensitivity in relation to $A B C B 1$ expression, efflux and single nucleotide polymorphisms in ovarian cancer}

Bo Gao ${ }^{1,2}$, Amanda Russell ${ }^{3}$, Jonathan Beesley ${ }^{4}$, Xiao Qing Chen ${ }^{4}$, Sue Healey ${ }^{4}$, Michelle Henderson ${ }^{3}$, Mark Wong ${ }^{5}$, Catherine Emmanuel ${ }^{1,2}$, Laura Galletta ${ }^{6}$, Sharon E. Johnatty ${ }^{4}$, David Bowtell ${ }^{6,7,8,9}$, Australian Ovarian Cancer Study Group*, Michelle Haber ${ }^{3}$, Murray Norris ${ }^{3}$, Paul Harnett ${ }^{2,5}$, Georgia Chenevix-Trench ${ }^{4}$, Rosemary L. Balleine $2,5,10 \dagger \&$ Anna deFazio ${ }^{1,2,5} \dagger$

${ }^{1}$ Department of Gynaecological Oncology, Westmead Hospital, ${ }^{2}$ Westmead Institute for Cancer Research, University of Sydney at Westmead Millennium Institute, Sydney, Australia, ${ }^{3}$ Children's Cancer Institute Australia, Sydney, Australia, ${ }^{4}$ QIMR Berghofer Medical Research Institute, Brisbane, Australia, ${ }^{5}$ Crown Princess Mary Cancer Centre, Westmead Hospital, Westmead, Australia, ${ }^{6}$ Peter MacCallum Cancer Centre, East Melbourne, Australia, ${ }^{7}$ Sir Peter MacCallum Department of Oncology, ${ }^{8}$ Department of Pathology, University of Melbourne, Melbourne, Australia, ${ }^{9}$ Department of Biochemistry and Molecular Biology, University of Melbourne, Melbourne, Australia, ${ }^{10}$ Pathology West ICPMR Westmead, Sydney, Australia.

ABCB1 (adenosine triphosphate-binding cassette transporter B1) mediates cellular elimination of many chemotherapeutic agents including paclitaxel, which is commonly used to treat ovarian cancer. A significant association between common single nucleotide polymorphisms (SNPs) in $A B C B 1$ and progression-free survival has been reported in patients with ovarian cancer. Variable paclitaxel clearance due to genotype specific differences in ABCB1 activity in cancer cells and/or normal tissues may underlie the association. Using cell-based models, we evaluated the correlations between $A B C B 1$ expression, polymorphisms, transporter activity and paclitaxel sensitivity in ovarian cancer $(n=10)$ and lymphoblastoid $(n=19)$ cell lines. Close associations between $A B C B 1$ expression, transporter function and paclitaxel sensitivity were found in lymphoblastoid cell lines, although we could not demonstrate an association with common SNPs. In ovarian cancer cell lines, $A B C B 1$ expression was low and the association between expression and function was lost. These results suggest that $A B C B 1$ related survival difference in ovarian cancer patients is more likely to be due to differential whole body paclitaxel clearance mediated by normal cells rather than a direct effect on cancer cells.

varian cancer is the seventh leading cause of cancer mortality among women globally. Most patients are treated with paclitaxel and carboplatin chemotherapy after surgery ${ }^{1}$. However, chemotherapy response varies widely between individuals. While a number of factors including tumor histology, somatic mutations, environmental and drug-drug interactions, can contribute to the inter-individual variability, inherited genetic variations are increasingly understood to influence drug disposition and activity.

Polymorphisms in genes encoding drug transporter proteins are among the most clinically important genotypic variations that may impact on therapeutic agents ${ }^{2}$. The adenosine triphosphate (ATP)-binding cassette (ABC) transporter, ABCB1, also known as permeability-glycoprotein (P-gp) or multidrug resistance protein 1 (MDR1), is expressed in normal barrier tissues such as the apical membrane of intestine, the canalicular site of hepatocytes, tubular cells of the kidney, lymphocytes, and also various human cancers ${ }^{3}$. It mediates the rapid exit of a vast spectrum of therapeutic agents from cells including the chemotherapeutic drug paclitaxel ${ }^{4}$. A number of studies have explored the potential of using $A B C B 1$ polymorphisms as a predictive and/or prognostic marker in ovarian cancer, but no compelling or consistent results have been identified ${ }^{5-7}$. Several factors including patient numbers, study design and analytical methods may cause this discrepancy. In a cohort of 309 patients from the Australian Ovarian Cancer Study (AOCS), we found three common single nucleotide polymorphisms (SNPs) in ABCB1 (C1236T (rs1128503), G2677T/A (rs2032582) and C3435T (rs1045642)) were associated with significant differences in progression-free survival (PFS) for the subgroup of women with $\leq 1 \mathrm{~cm}$ residual disease who received paclitaxel and carboplatin first-line chemotherapy. In this subgroup of patients, PFS was significantly longer for individuals who carried the minor T/A alleles at the $2677 \mathrm{G}>\mathrm{T} / \mathrm{A}$ compared with homozygote GG 
carriers $(\mathrm{HZ}=0.61,95 \% \text { CI } 0.45-0.83 ; p=0.002)^{8}$. A similar effect was demonstrated in 281 patients from Scottish Randomised Trial in Ovarian Cancer 1 (SCOTROC1) who had no residual disease after surgery and received carboplatin and paclitaxel or docetaxel combination chemotherapy ${ }^{8}$. On the basis of these findings, we hypothesised that the association between $A B C B 1$ genotype and outcome in ovarian cancer may reflect differential cellular transport of paclitaxel in normal and/or cancer cells.

To investigate this, we used cell-based models to evaluate the associations between $A B C B 1$ genetic polymorphisms, gene expression, transporter activity and paclitaxel sensitivity in both ovarian cancer cell lines and lymphoblastoid cell lines (LCLs) derived from women with ovarian cancer. We reasoned that ovarian cancer cells have multiple and varied genomic and phenotypic alterations that may outweigh any effect of inherited polymorphisms, whereas phenotype-genotype associations may be better appreciated in normal cells such as lymphoblastoid cell lines.

\section{Results}

$A B C B 1$ expression was variable but higher in lymphoblastoid cell lines compared with ovarian cancer cells. Relative levels of $A B C B 1$ mRNA expression were determined in 19 LCLs and 10 ovarian cancer cell lines. There was a 20 -fold inter-individual variation in $A B C B 1$ expression in LCLs (Figure 1a). $A B C B 1$ expression was also variable in ovarian cancer cells although the level of $A B C B 1$ expression in these cells was very low (Figure 1b).

LCLs eliminated paclitaxel faster than ovarian cancer cell lines. To examine ABCB1 transporter activity, cellular bodipy-FL-paclitaxel accumulation and efflux were measured in 13 LCLs and six ovarian cancer cell lines selected to cover the spectrum of $A B C B 1$ expression and paclitaxel sensitivity, using flow cytometry. For these experiments, a range of concentrations of bodipy-FL-paclitaxel was tested and $0.1 \mu \mathrm{M}$ was selected as it allowed rapid accumulation and a steady state level of bodipy-FL-paclitaxel that was able to be measured reproducibly by flow cytometry, but had no obvious effect on cell viability during the short exposure time. Following incubation with $0.1 \mu \mathrm{M}$ bodipy-FL-paclitaxel, intracellular fluorescence activity reached a steady state in both cell types after 30 minutes (Supplementary Figure 1a). Under these conditions, we found no difference in median intracellular bodipy-FL-paclitaxel fluorescence activity between LCLs and ovarian cancer cells (Figure 2a).

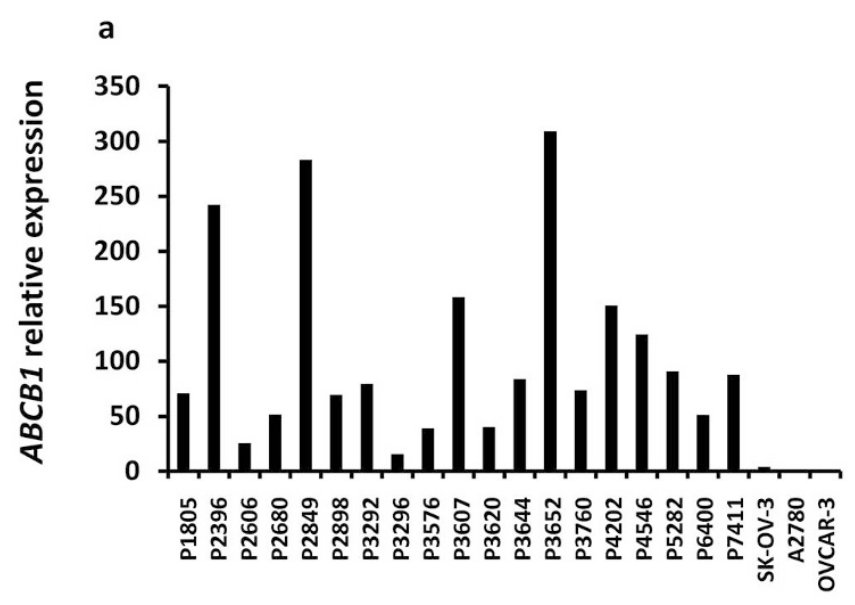

Cellular bodipy-FL-paclitaxel fluorescence intensity decreased over the efflux period (Figure 2b) and exhibited a three-phase elimination pattern - an initial rapid efflux phase in the first three to five minutes, followed by a slower efflux phase up to 15 minutes, usually reaching a plateau phase between 15 to 30 minutes (Supplementary Figures $1 \mathrm{~b} \& 1 \mathrm{c}$ ). The presence of an $\mathrm{ABC}$ transporter blocker (verapamil) significantly reduced bodipy-FL-paclitaxel efflux rate in all cell lines, both LCLs $(n=13)$ and ovarian cancer cell lines $(n=6)$, providing supporting evidence that bodipy-FL-paclitaxel efflux was mediated by the ABCB1 transporter (representative examples shown in Supplementary Figures 1d-1f).

A fitted curve and an efflux constant $\mathrm{K}_{\mathrm{E}}$ were generated for each cell line based on a three compartment model using the solver function of Microsoft Excel (Figure 2c). The median efflux $\mathrm{K}_{\mathrm{E}}$ was significantly higher in LCLs compared to ovarian cancer cell lines $(0.470$ vs $0.124, p=0.011$ ) in an independent median test, indicating more rapid efflux of paclitaxel from LCLs (Figure $2 \mathrm{~d}$ ). The range of variation in efflux constant $K_{\mathrm{E}}$ in LCLs (3.6-fold) and ovarian cancer cell lines (3.2-fold) was similar.

$A B C B 1$ expression was correlated with paclitaxel accumulation and efflux in LCLs. The relationships between $A B C B 1$ mRNA relative expression, bodipy-FL-paclitaxel accumulation and efflux constant $\mathrm{K}_{\mathrm{E}}$ were assessed in a correlation matrix by Spearman or Pearson correlation tests depending on data distribution. In LCLs, there was an inverse correlation between $A B C B 1$ expression and intracellular bodipy-FL-paclitaxel accumulation $(\mathrm{Rs}=-0.571, p$ $=0.041$ ), and a positive correlation between $A B C B 1$ expression and the efflux constant $\mathrm{k}_{\mathrm{E}}(\mathrm{Rs}=0.593, p=0.033)$ (Table 1). Paclitaxel accumulation correlated strongly with efflux $(\mathrm{R}=0.748$, $p=0.003$ ). These results indicated that LCLs expressing higher levels of $A B C B 1$ accumulated less intracellular paclitaxel and eliminated paclitaxel more efficiently.

In contrast, there was no correlation between $A B C B 1$ expression and paclitaxel accumulation or efflux in ovarian cancer cell lines (Table 1).

Ovarian cancer cell lines were more resistant to paclitaxel with a wider variation in $\mathbf{I C}_{\mathbf{5 0}}$. The cytotoxicity of paclitaxel was measured in 19 LCLs and 10 ovarian cancer cell lines (Figure 3). There was a 10fold variation in paclitaxel $\mathrm{IC}_{50}$ among ovarian cancer cell lines. PEO14 was the most paclitaxel resistant cell line with an $\mathrm{IC}_{50}$ of $15.0 \mathrm{nM}$, while CAOV-3 the most sensitive with a $\mathrm{IC}_{50}$ of $1.5 \mathrm{nM}$.

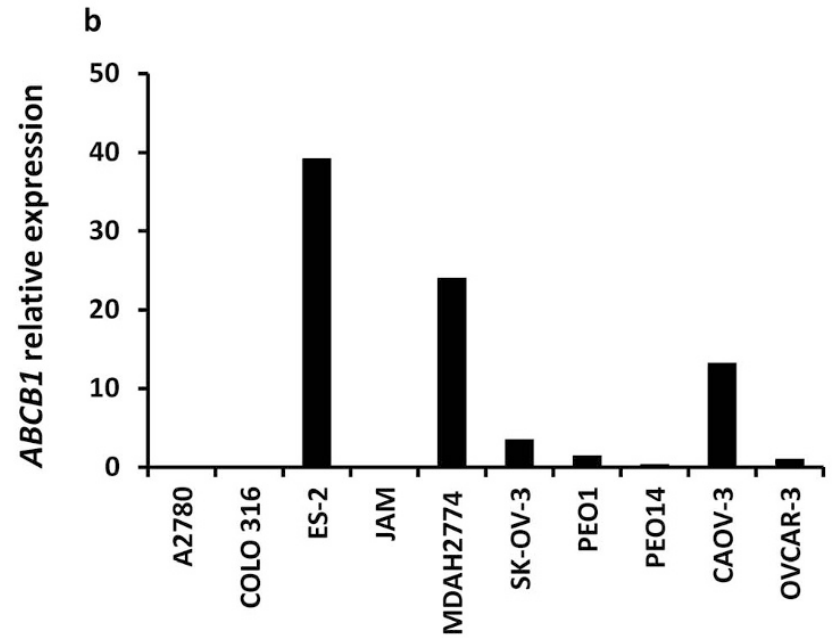

Figure $1 \mid$ Relative $A B C B 1$ expression in lymphoblastoid and ovarian cancer cell lines. $A B C B 1$ mRNA expression was measured by quantitative RT-PCR in (a) 19 lymphoblastoid cell lines and (b) 10 ovarian cancer cell lines. All results were expressed relative to the level expressed in OVCAR-3. The expression in SK-OV-3, A2780 and OVCAR-3 in (a) were measured in parallel to compare the levels of relative expression between lymphoblastoid cell lines and ovarian cancer cell lines. The results presented were the geometric mean of values normalized to the three control genes HPRT, GUSB and PGK1. 


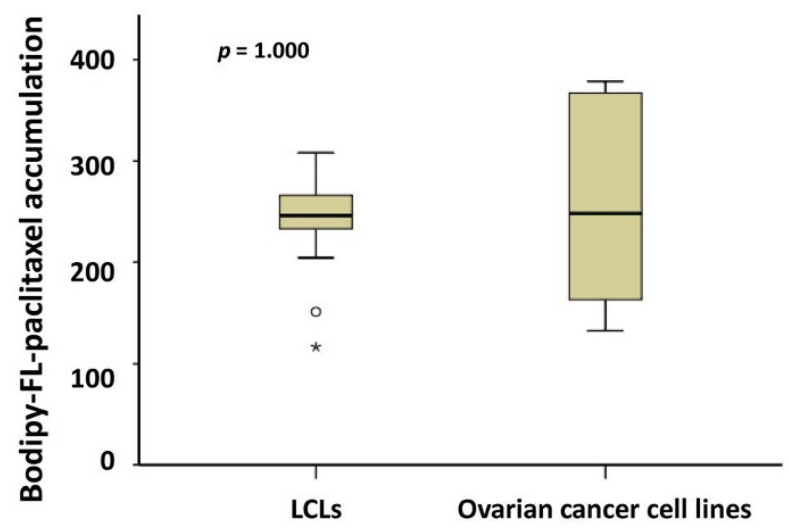

c

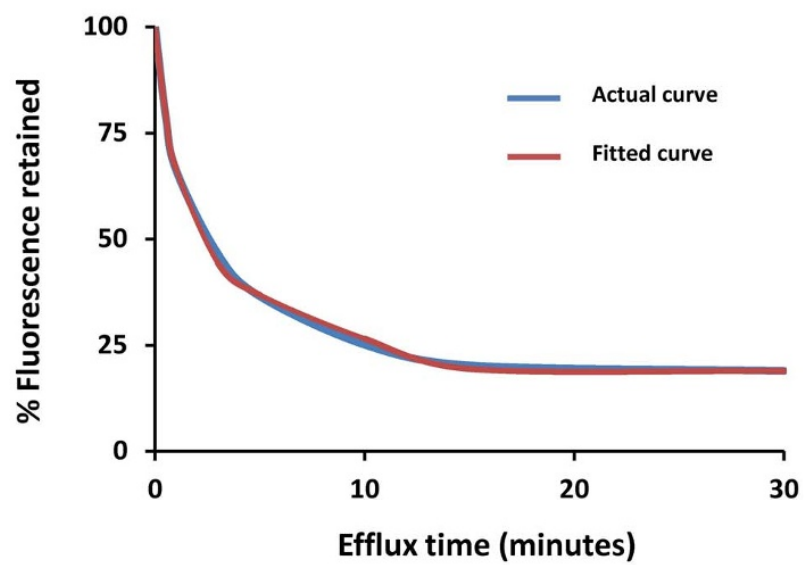

b

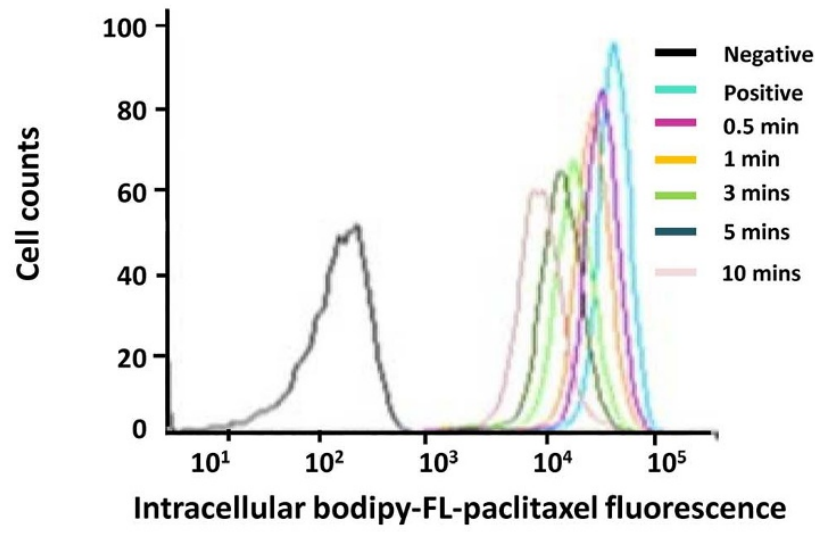

d

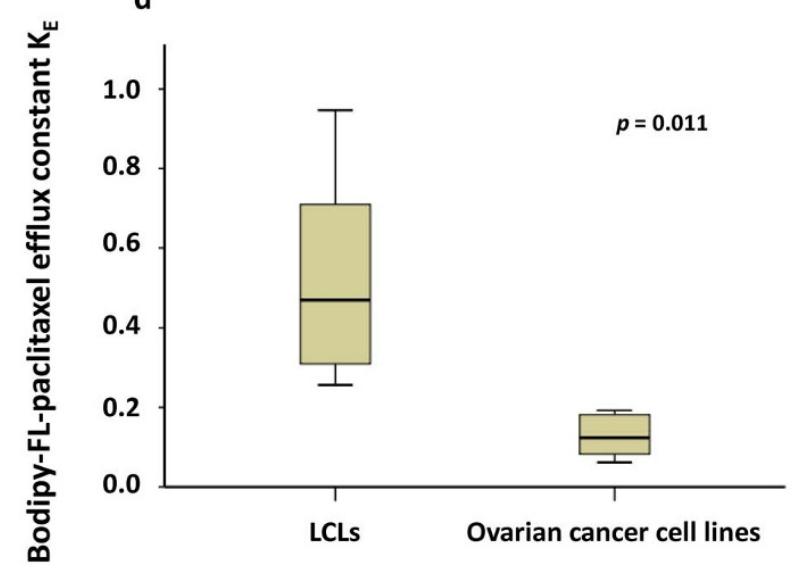

Figure $2 \mid$ Cellular accumulation and efflux of bodipy-FL-paclitaxel, a fluorescent ABCB1 substrate in lymphoblastoid and ovarian cancer cell lines measured by flow cytometry. (a) Median bodipy-FL-paclitaxel accumulated in 13 LCLs and six ovarian cancer cell lines, by independent-samples median test; (b) Decreasing bodipy-FL-paclitaxel fluorescence intensity over a 10 minutes efflux time, in one LCL as a representative example; (c) A fitted curve generated by Solve function of Excel as compared to the actual curve of percentage fluorescence retained, in one LCL as a representative example;

(d) Median efflux constant $K_{E}$ in LCLs and ovarian cancer cell lines, by independent-samples median test. All results are mean of at least two independent experiments.

LCLs were generally more sensitive to paclitaxel and had a much smaller (2.4-fold) variation in paclitaxel $\mathrm{IC}_{50}$, ranging from 2.9 to $6.8 \mathrm{nM}$.

Paclitaxel toxicity correlated with efflux in lymphoblastoid cell lines. In simple linear regression analysis, there was a trend of correlation between $A B C B 1$ expression and paclitaxel $\mathrm{IC}_{50}(\mathrm{r}=$ 0.429, $p=0.067$ ) (Figure $4 \mathrm{a}$ ) and a trend of inverse correlation between paclitaxel accumulation and toxicity $(\mathrm{r}=-0.546, p=$
0.053) (Figure 4c) in LCLs. The correlation between paclitaxel efflux constant $\mathrm{k}_{\mathrm{E}}$ and paclitaxel $\mathrm{IC}_{50}$ was strongly positive $(\mathrm{r}=$ 0.819, $p=0.001$ ) (Figure 4e), demonstrating that LCLs that eliminated paclitaxel more efficiently were more resistant to paclitaxel. In multiple linear regression analysis, paclitaxel efflux constant $\mathrm{K}_{\mathrm{E}}$ was the only independent variable strongly correlating with paclitaxel $\mathrm{IC}_{50}$ (Beta coefficient $\left.=0.945, p=0.009\right)$ (Table 2).

There were no associations between paclitaxel cytotoxicity and accumulation or efflux in ovarian cancer cell lines (Figures $4 \mathrm{~b}, 4 \mathrm{~d}$

Table 1 | Correlation matrix between $A B C B 1$ relative expression, bodipy-FL-paclitaxel accumulation and efflux constant $K_{E}$ in lymphoblastoid cell lines and ovarian cancer cell lines using Spearman or Pearson correlation test

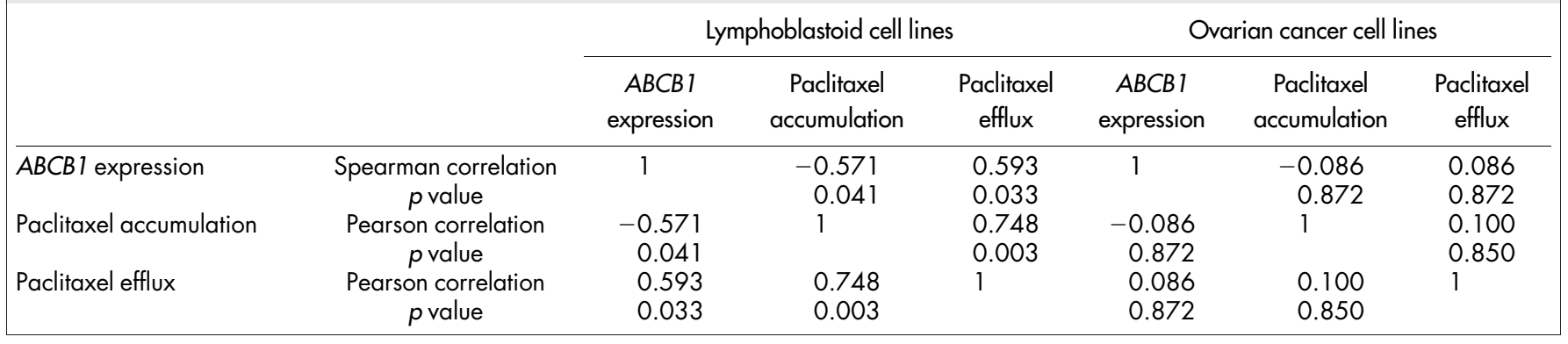


a

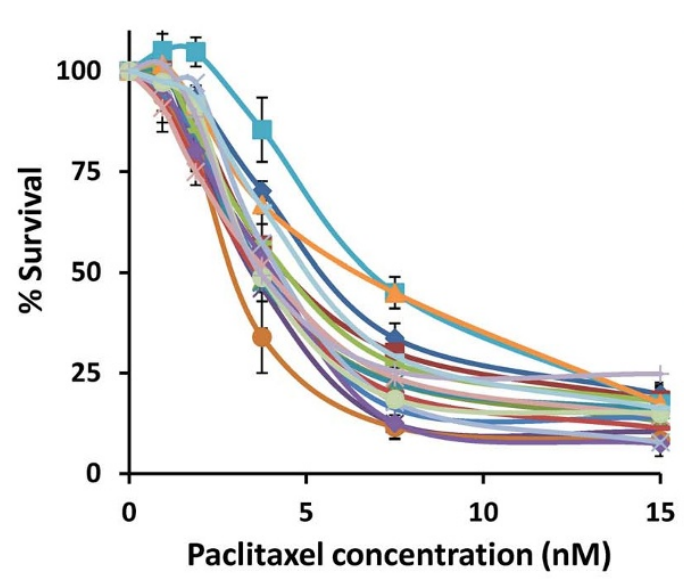

b

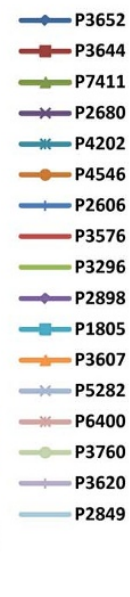

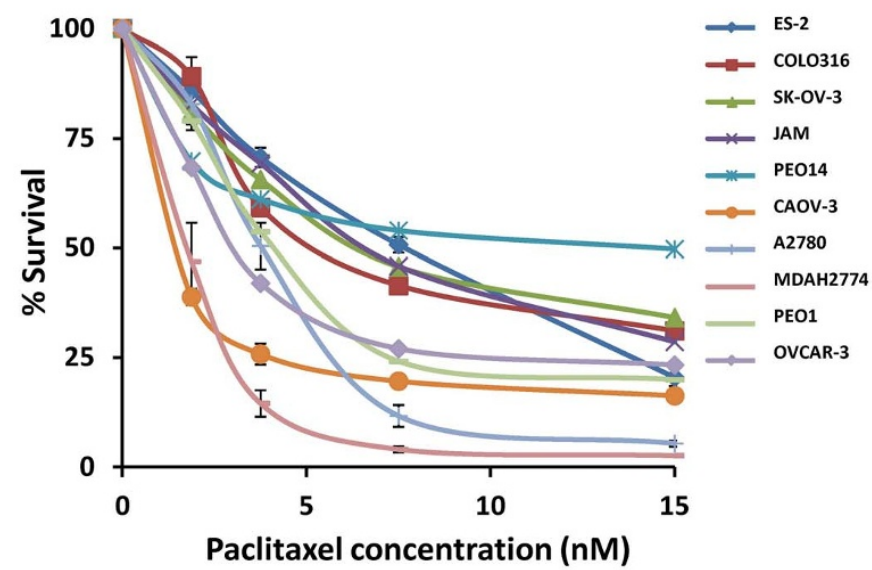

Figure 3 Inhibition of proliferation by paclitaxel in lymphoblastoid and ovarian cancer cell lines. Effect of paclitaxel on cell proliferation was measured in (a) 19 lymphoblastoid cell lines and (b) 10 ovarian cancer cell lines using the CellTitre AQueous One Solution Cell Proliferation Assay, measured after $72 \mathrm{hrs}$ drug exposure, represented as a percentage of vehicle treated control. Results are mean with standard error of mean of three independent experiments performed in triplicate.

and $4 \mathrm{f}$ and Table 2). Exploratory analyses were performed in three ovarian cancer cell lines with relatively high $A B C B 1$ expression (ES-2, MDAH2774 and CAOV-3). Although there was trend of correlation between $A B C B 1$ expression and paclitaxel $\mathrm{IC}_{50}$, it was not statistically significant $(p=0.206)$. There was no association between efflux, accumulation and paclitaxel $\mathrm{IC}_{50}$, but the very small sample number $(n=3)$ limited the interpretation of these results.

No associations between $A B C B 1$ genotype and paclitaxel cytotoxicity or $A B C B 1$ expression and transporter activity were identified. Common $A B C B 1$ SNPs were examined in all cell lines. No associations were observed between SNPs in $A B C B 1$ exon 12 (C1236T), 21 (G2677T/A) and 26 (C3435T) and $A B C B 1$ expression (Supplementary Figure 2), paclitaxel accumulation, efflux or cytotoxicity in LCLs or ovarian cancer cell lines (Jonckheere-Terpstra test for ordered alternatives) (Figure 5).

\section{Discussion}

Several studies have suggested an association between $A B C B 1$ genotypes and clinical outcome in cancer patients ${ }^{9-11}$, including ovarian cancer $^{5,8,11,12}$. To investigate whether this association reflected differences in ABCB1 mediated transport of paclitaxel in normal or ovarian cancer cells, we compared $A B C B 1$ expression, paclitaxel efflux, cytotoxicity and polymorphisms in normal and ovarian cancer cell lines under the same experimental conditions. In LCLs there was a clear association between $A B C B 1$ expression levels, the rate of paclitaxel efflux and cytotoxicity. ABCB1 is responsible for the apical transport of various chemotherapeutic agents like paclitaxel. The degree of expression and the functionality of the transporter can directly affect the intracellular concentration of paclitaxel, therefore its growth inhibitor effects. As a result, more efficient efflux and higher relative $A B C B 1$ expression can lead to higher paclitaxel $\mathrm{IC}_{50}$. In contrast, $A B C B 1$ expression in ovarian cancer cells was very low and not associated with paclitaxel efflux or cytotoxicity.

$\mathrm{ABCB} 1$ transporters are expressed and functionally active in all peripheral blood cells, except in granulocytes ${ }^{13}$. The efficient efflux of chemotherapeutic agents in peripheral blood and haematopoietic cells may confer a degree of protection to these cell types. This finding is consistent with the observations of pioneers such as Skipper and Schabel, who found that some cancer cells recovered more slowly (or not at all) compared to the more robust non-cancer cells when incubated together in a high concentration of cytotoxic drugs for hours or days, followed by longer periods of recovery ${ }^{14}$.
We found no apparent association between $A B C B 1$ SNPs and expression, efflux or cytotoxicity in LCLs. This is in contrast with the study of 31 healthy Caucasian volunteers by Hitzl et al., which reported a significant correlation between $A B C B 1$ genotypes at position 3435 with the efflux of rhodamine, an ABCB1 substrate, from freshly isolated CD56 ${ }^{+}$natural killer cells ${ }^{15}$. They also demonstrated a correlation between genotype and mRNA expression in leukocytes. Specifically these investigators reported that subjects who were homozygous CC at position 3435 had faster rhodamine efflux from $\mathrm{CD}^{+} 6^{+}$natural killer cells and higher $A B C B 1$ mRNA expression in leukocytes than subjects with the TT genotype. More recently Wong et al. reported that $A B C B 1$ genotype was associated with hepatic elimination of ABCB1 substrate technetium Tc99m-labeled sestamibi in a cohort of cancer patients ${ }^{16}$. In their study, patients with CC genotype at position 3435 of $A B C B 1$ gene had faster hepatic sestamibi clearance than TT genotype. These data indicated potential genotype related differences in ABCB1 function, which are consistent with our clinical findings in ovarian cancer patients showing women with CC genotype at position 3435 had worse PFS $^{8}$.

Limitations of this study to detect an association between $A B C B 1$ genotype and paclitaxel effect in normal cells include low levels of absolute $A B C B 1$ expression in LCLs, compared to liver and bowel; the relatively small number of LCLs examined $(n=19)$, and the potential impact of exogenous factors during the process of EBVtransformation and in vitro growth that may modify the phenotypes compared with in vivo analyses.

$\mathrm{ABCB} 1$ is over-expressed in tumors that originate from tissues expressing physiologically high levels of ABCB1 such as adrenal gland, kidney, liver and colon ${ }^{17}$. In cell lines derived from these tumors, an inverse correlation between $A B C B 1$ expression and sensitivities to chemotherapeutic drugs that are substrates of ABCB1 transport has been observed ${ }^{18-20}$. Consistent with the reported low level expression in normal ovaries and untreated epithelial ovarian cancer $^{3,21}$, we found $A B C B 1$ was expressed at very low levels in the panel of 10 ovarian cancer cell lines. The presence of verapamil, an ABCB1 blocker, inhibited paclitaxel efflux in all ovarian cancer cell lines tested regardless of the level of $A B C B 1$ expression, suggesting ABCB1 transporter is functional in all ovarian cancer cell lines. Despite the lower levels of $A B C B 1$ expression and less efficient paclitaxel elimination, some ovarian cancer cell lines were more resistant to paclitaxel than LCLs. Ovarian cancer cells have multiple genomic alterations and the loss of association between $A B C B 1$ expression, paclitaxel elimination and paclitaxel resistance could 

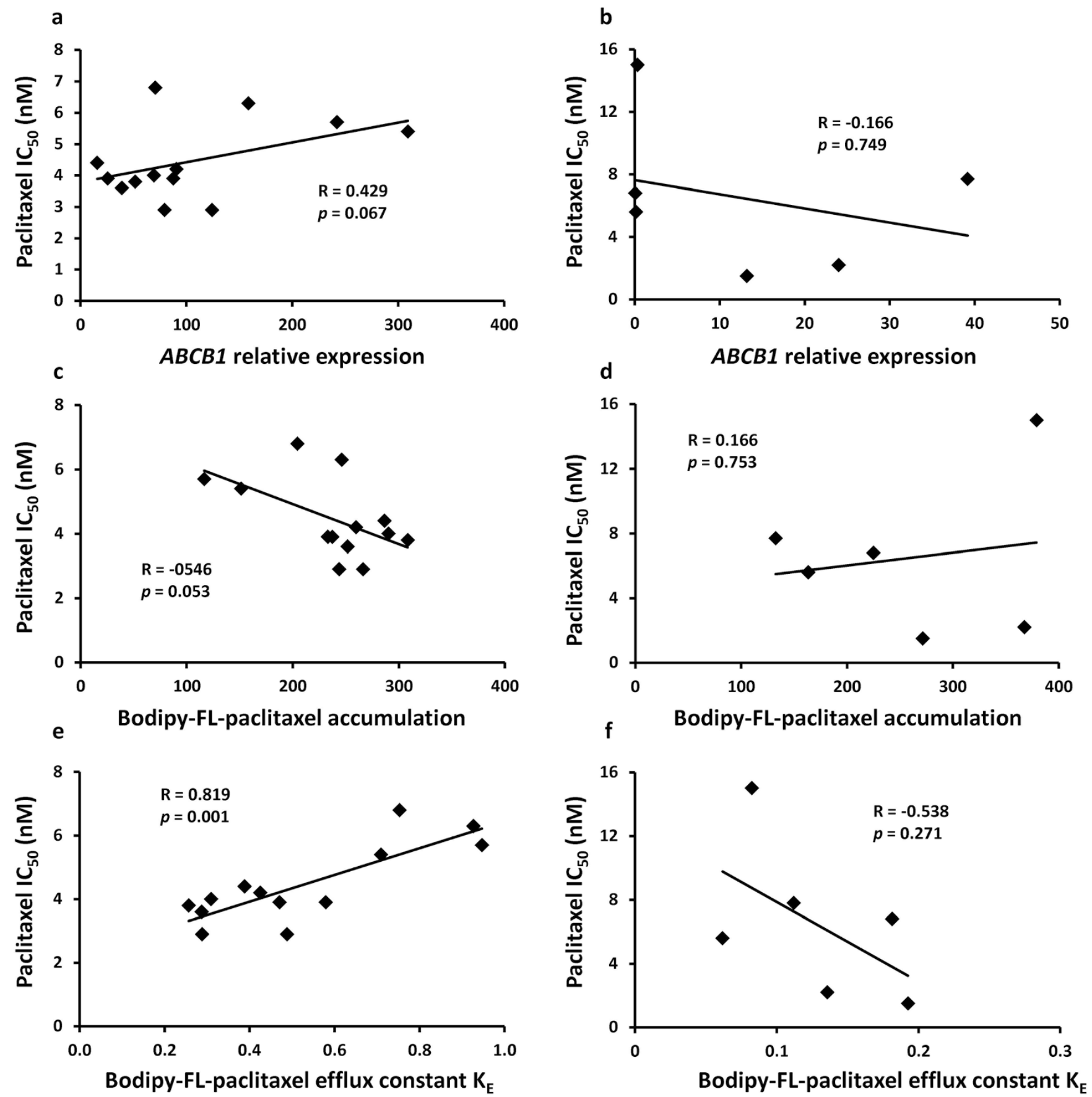

Figure $4 \mid$ Relationship between growth inhibitory effects of paclitaxel, $A B C B 1$ expression and cellular drug accumulation and efflux. Paclitaxel growth inhibitory effect ( $\mathrm{IC}_{50}$ ) was determined in 13 lymphoblastoid cell lines (a), (c) and (e) and 6 ovarian cancer cell lines (b), (d) and (f) by CellTitre AQueous One Solution Cell Proliferation Assay after 72 hrs drug exposure. ABCB1 mRNA expression (a) and (b) was measured by RT-PCR and bodipy-FLpaclitaxel accumulation (c) and (d) and efflux (e) and (f) were measured by flow cytometry. The correlation between paclitaxel IC ${ }_{50}$ and $A B C B 1$ expression (a) and (b), paclitaxel $\mathrm{IC}_{50}$ and drug accumulation (c) and (d) and the correlation between $\mathrm{IC}_{50}$ and drug efflux constant $\mathrm{K}_{\mathrm{E}}(\mathrm{e})$ and (f) were determined by simple linear regression analysis.

Table 2 | Multiple linear regression analysis of $A B C B 1$ expression, bodipy-FL-paclitaxel accumulation, efflux and paclitaxel $I_{50}$

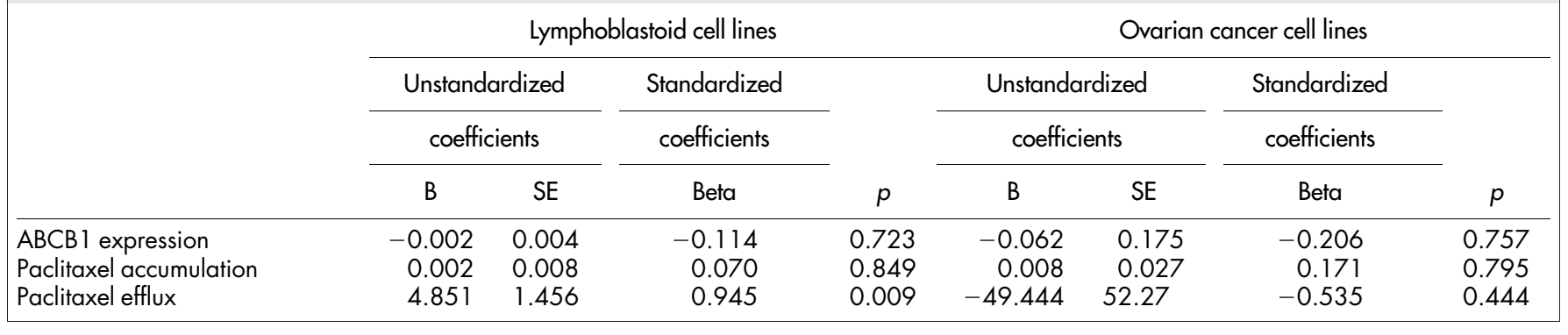



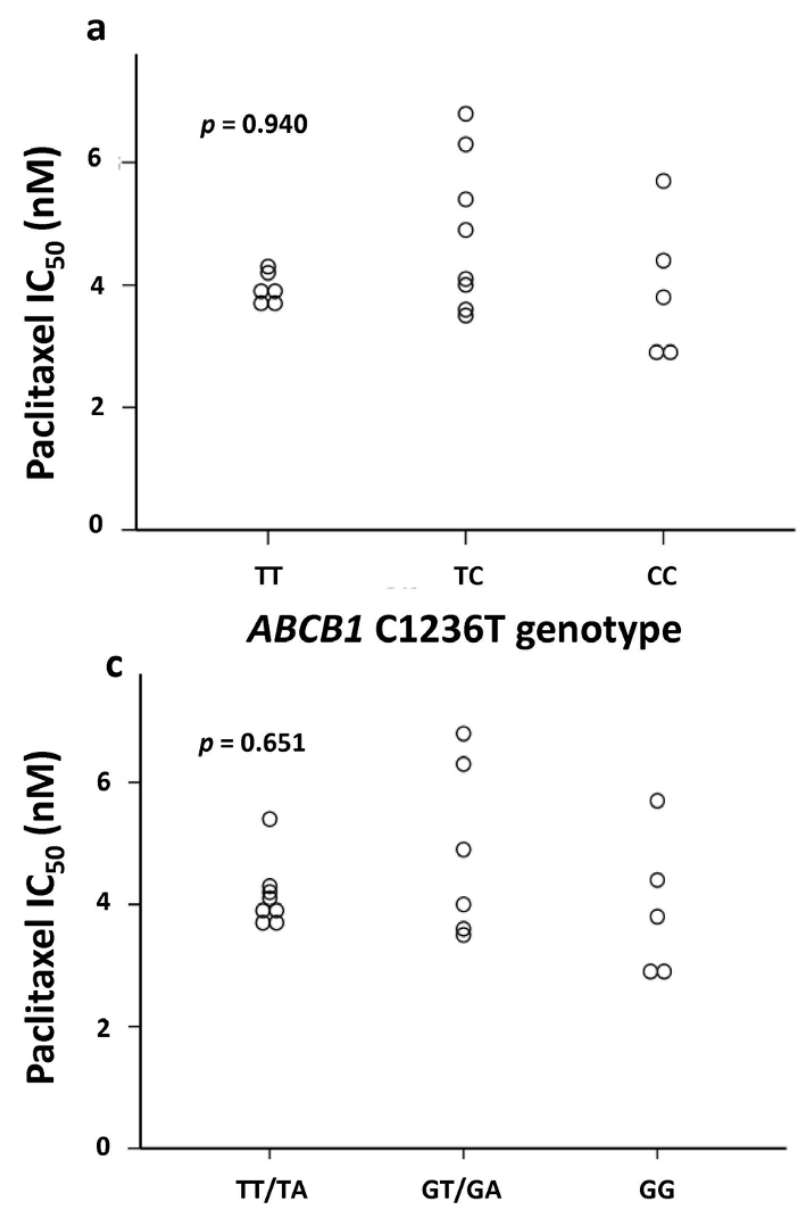

ABCB1 G2677T>A genotype

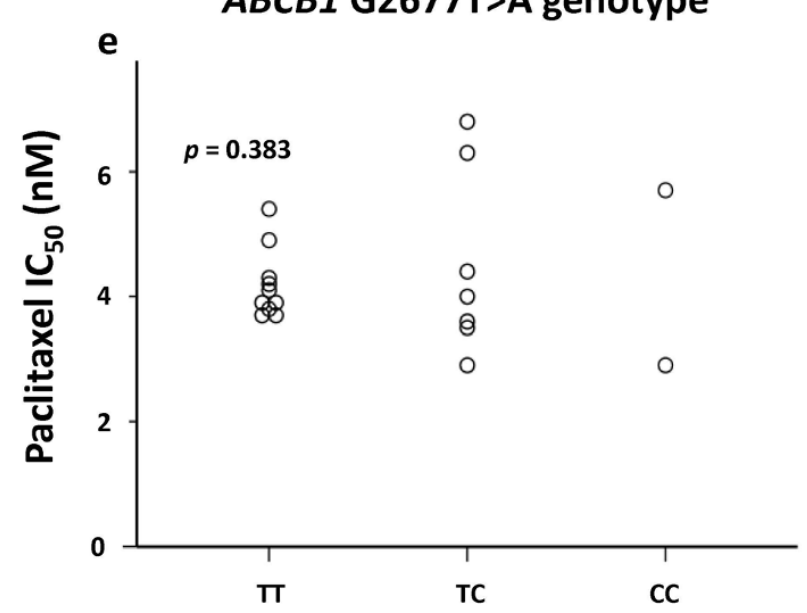

ABCB1 C3435T genotype
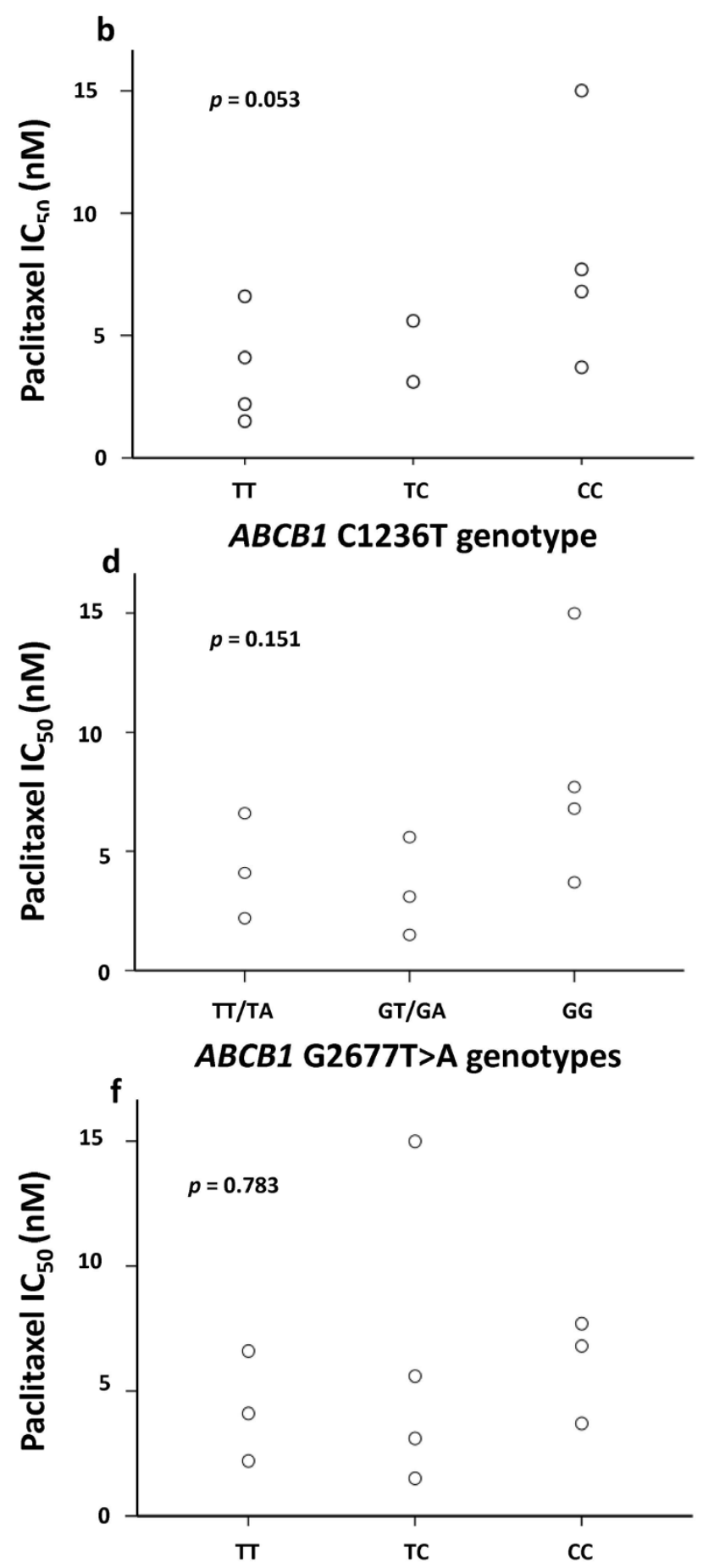

$A B C B 1$ C3435T genotypes

Figure $5 \mid$ Association between growth inhibitory effects of paclitaxel and $A B C B 1$ genotypes. Paclitaxel growth inhibitory effects (IC ${ }_{50}$ ) were determined in 19 lymphoblastoid cell lines (a), (c) and (e) and 10 ovarian cancer cell lines (b), (d) and (f), by CellTitre AQueous One Solution Cell Proliferation Assay after 72 hrs drug exposure. ABCB1 genotype was determined by iPLEX genotyping on Sequenom's MassARRAY platform. Associations between $\mathrm{IC}_{50}$ and $A B C B 1$ genotypes at C1236T (a) and (b), G2677T/A (c) and (d) and C3435T (e) and (f) were analysed by JonckheereTerpstra test.

be due to a variety of factors including somatic mutations ${ }^{22}$, oncogene expression and amplification or inappropriate growth factor regulation ${ }^{23}$ in ovarian cancer cells. Other factors that could potentially contribute to paclitaxel resistance despite low $A B C B 1$ expression in ovarian cancer cells, include alterations in tubulin binding and dynamics due to tubulin mutation ${ }^{24}$, altered expression of microtubule-associated proteins (MAP) $)^{25}$ and decreased sensitivity to apoptosis-induced stimuli.

Genomic differences between cancer cell lines and tissue samples have been pointed out in several studies ${ }^{22,26,27}$. So primary ovarian cancer cells, freshly isolated from either solid tumor or malignant ascites, may offer an experimental system that resembles the patient's 
situation more closely. The ovarian cancer cell lines used in this study were derived from tumors of various histological subtypes and carry a variety of mutations ${ }^{22}$. Restricting analyses to cell lines with a similar histological/genomic profile may yield a different result.

Although our study did not confirm the presence of an $A B C B 1$ genotype-phenotype correlation in LCLs or ovarian cancer cell lines, the strong association we found between $A B C B 1$ expression, transporter function and paclitaxel cytotoxicity in LCLs, together with the very low level $A B C B 1$ expression in the panel of 10 ovarian cancer cell lines, suggests that the observed $A B C B 1$ genotype-related outcome differences in ovarian cancer patients is more likely to be due to differential, whole body elimination of paclitaxel rather than a direct effect on cancer tissues. Few studies published to date have assessed the effects of $A B C B 1$ genotypes on paclitaxel clearance in ovarian cancer patients $s^{7,28,29}$, and the results have been contradictory. Possible reasons include small patient numbers and significant variation in paclitaxel dosage and infusion time. Our results suggest that further detailed assessment of the effect of SNPs in $A B C B 1$, and potentially SNPs in other genes on paclitaxel clearance and ultimate association with ovarian cancer survival is warranted.

\section{Methods}

Cell line authentication and culture conditions. EBV-transformed Blymphoblastoid cell lines (LCLs) from 19 Caucasian ovarian cancer patients were obtained through the Australian Ovarian Cancer Study (AOCS) ${ }^{30}$. Human ovarian cancer cell lines A2780, COLO316, JAM, PEO1, PEO14, OVCAR-3 (Professor Georgia Chenevix-Trench, QIMR Berghofer Medical Research Institute, Australia), CAOV-3, ES-2 and SK-OV-3 (ATCC, Manassas, VA, USA) were authenticated by STR profiling (Promega Cell ID System, Promega Corporation, Madison WI, USA) against published profiles. MDAH2774 was profiled, but no reference was available for comparison.

LCLs and ovarian cancer cells were maintained in RPMI 1640 (Invitrogen Corporation, Carlsbad CA, USA) supplemented with $15 \%$ and $10 \%$ fetal calf serum (SAFC Biosciences, Lenexa KS, USA) v/v respectively. All cells were grown at $37^{\circ} \mathrm{C}$ in a humidified atmosphere containing $5 \% \mathrm{CO}_{2}$. Cells were tested regularly for Mycoplasma contamination and shown to be negative.

This study was approved by the Western Sydney Local Health District and Queensland Institute of Medical Research Human Research Ethics Committees, and AOCS was approved by the Human Research Ethics Committees at the Peter MacCallum Cancer Centre, Queensland Institute of Medical Research, University of Melbourne and all participating hospitals. Methods were carried out in accordance with the approved guidelines and informed consent was obtained from all subjects.

$A B C B 1$ genotyping. Genomic DNA was extracted using DNeasy Blood \& Tissue Kit (Qiagen, Hilden, Germany). Common ABCB1 SNPs at exon 12 (C1236T), 21 (G2677T/A) and 26 (C3435T) were examined by iPLEX genotyping on Sequenom's MassARRAY platform ${ }^{8}$.

Expression of $A B C B 1 \mathrm{mRNA}$ by real-time PCR. RNA was extracted using the RNeasy Mini Kit (Qiagen GmbH, Hilden, Germany). RNA was reverse transcribed with MMLV reverse transcriptase (Life Technologies, Carlsbad CA, USA) as previously described ${ }^{31}$. Real-time PCR quantitation of gene expression utilising TaqMan ${ }^{\circledR}$ gene expression assays ( $A B C B 1$, assay ID Hs $00184500 \mathrm{~m} 1 ;$ GUSB, assay ID 4326320E; HPRT, assay ID 4326321E; PGK1, assay ID 4326318E) were conducted using a 7900HT Fast Real-Time PCR system (Applied Biosystems, Foster City CA, USA). Gene expression levels were determined using the $\Delta \Delta \mathrm{Ct}$ method and expressed relative to a calibrator. Expression values were calculated as the geometric mean of values normalized to the three control genes HPRT, GUSB and PGK1.

Paclitaxel growth inhibition assay. Drug concentrations that inhibited $50 \%$ of cell growth $\left(\mathrm{IC}_{50}\right)$ were determined using the CellTitre AQueous One Solution Cell Proliferation Assay (Promega, Madison WI, USA). Briefly, cell suspensions (100 $\mu \mathrm{L}$, 5000 cells for LCLs and 10000 cells for cancer cell lines) were plated in 96-well flat bottom plates. After 24-h incubation, cells were treated with $50 \mu \mathrm{l}$ of increasing concentrations (0-15 nM) of paclitaxel injection solution (Sigma-Aldrich Inc, St Louis MA, USA) and vehicle control (Cremophor $\mathrm{EL}^{\mathrm{TM}}$ and dehydrated ethanol USP $(1: 1, \mathrm{v} / \mathrm{v}))$ for 72 hours before adding $30 \mu \mathrm{L}$ of MTS reagent. Following $3-\mathrm{h}$ incubation, absorbance at $490 \mathrm{~nm}$ was recorded on a Wallac Victor ${ }^{2} 1420$ Multilabel Counter (Perkin Elmer Life Sciences, Waltham, MA, USA). Percent survival was quantified according to the manufacturer's protocol. Paclitaxel $\mathrm{IC}_{50}$ was determined by curve fitting of percentage cell survival against concentrations of paclitaxel. All experiments were conducted at least three experiments in triplicate.

Bodipy-FL-paclitaxel accumulation and efflux assay. To allow bodipy-FL-paclitaxel (Invitrogen, Carlsbad CA, USA) accumulation, cell suspensions at densities of $10^{6}$ cells per $\mathrm{mL}$ were incubated with bodipy-FL-paclitaxel at a concentration of $0.1 \mu \mathrm{M}$ for 30 minutes at $37^{\circ} \mathrm{C}$ and $5 \% \mathrm{CO}_{2}$. After accumulation, cells were aliquoted into
Eppendorf tubes (Eppendorf, Hamburg, Germany) (1 mL per tube) on ice, centrifuged for 5 minutes at $400 \mathrm{~g}$ at $4^{\circ} \mathrm{C}$ and washed twice with ice old PBS to remove extracellular bodipy-FL-paclitaxel.

After washing, the positive control sample was resuspended in $1 \mathrm{~mL}$ ice-cold medium for the measurement of the baseline fluorescence using a FACSCanto flow cytometer (488 nm, $20 \mathrm{~mW}$ laser; 530/30 band pass filter) (BD Biosciences, Franklin Lakes, New Jersey, USA). The remaining samples were resuspended in $1 \mathrm{~mL} 37^{\circ} \mathrm{C}$ medium and incubated at $37^{\circ} \mathrm{C}$ for $0.5,1,3,5,10,15$ and 30 minutes respectively to allow paclitaxel efflux. Three samples were incubated in medium containing verapamil (Abbott Australasia, Botany, Australia) at a concentration of $1 \mu \mathrm{g} / \mathrm{mL}$ for 5, 10 and 15 minutes respectively.

Appropriate threshold values were set to exclude clumps and debris, and samples were gated on forward scatter vs side scatter to include lymphoblastoid or cancer cell populations. A minimum of 10,000 events were collected per sample. Data was expressed as median bodipy-FL-paclitaxel fluorescence. Bodipy-FL-paclitaxel fluorescence in control sample, after baseline dye uptake, was set as $100 \%$. Bodipy-FLpaclitaxel fluorescence in efflux samples, exposed to different efflux time periods, was used to calculate the percentage of bodipy-FL-paclitaxel retained in the cells in comparison with control.

Pharmacokinetic modelling of bodipy-FL-paclitaxel cellular elimination. Intracellular paclitaxel fluorescence intensity is the combined result of active efflux through $\mathrm{ABCB} 1$ transporter, passive diffusion/efflux through the cell membrane and redistribution of the fluorescence from medium into the cells at the late phase of efflux assay. Hence a three compartment model was developed to reflect the changes. The summation of the kinetics of intra-cellular fluorescence resulted in an exponential equation of the following form: percentage of intra-cellular fluorescence at time $t=$ $\mathrm{Ae}^{-\mathrm{kEt}}+\mathrm{Be}^{-\mathrm{kDt}}+\mathrm{Ce}^{-\mathrm{kRt}}$, where $\mathrm{A}, \mathrm{B}$ and $\mathrm{C}$ are constants; $\mathrm{k}_{\mathrm{E}}$ is the active efflux constant; $\mathrm{k}_{\mathrm{D}}$ is the passive diffusion/efflux constant and $\mathrm{k}_{\mathrm{R}}$ is the redistribution constant. This equation was derived from fluorescence data of cell lines by the use of the solver function of Microsoft Excel (Office XP; Microsoft Corp, Redmond, Wash, USA). The efflux constant $\left(\mathrm{k}_{\mathrm{E}}\right)$ was used as the measure of bodipy-FL-paclitaxel cellular efflux rate and ABCB1 transporter activity.

Statistical analysis. Spearman or Pearson correlation tests were used to assess the relationships between $A B C B 1$ expression, bodipy-FL-paclitaxel accumulation and efflux constant $\mathrm{K}_{\mathrm{E}}$. Linear regression analysis was performed to assess their impacts on paclitaxel $\mathrm{IC}_{50}$. Independent median test was used to compare the median bodipyFL-paclitaxel accumulation and efflux between LCLs and ovarian cancer cell lines. Nonparametric Jonckheere-Terpstra test was used to compare the distribution of $A B C B 1$ expression, paclitaxel cytotoxicity and bodipy-FL-paclitaxel efflux constant $\mathrm{k}_{\mathrm{E}}$ between cell lines of different genotypes. All calculations were performed with SPSS for Windows v21 (SPSS, Chicago, Ill, USA). All tests are two-tailed and p-values $\leq 0.05$ were considered to be of statistical significance.

1. Cannistra, S. A. Cancer of the ovary. The N Engl J Med 351, 2519-2529 (2004).

2. Bosch, T. M. Pharmacogenomics of drug-metabolizing enzymes and drug transporters in chemotherapy. Methods Mol Bio 448, 63-76 (2008).

3. Goldstein, L. J. et al. Expression of a multidrug resistance gene in human cancers. J Natl Cancer Inst 81, 116-124 (1989).

4. Cascorbi, I. Role of pharmacogenetics of ATP-binding cassette transporters in the pharmacokinetics of drugs. Pharmacol Ther 112, 457-473 (2006).

5. Green, H., Soderkvist, P., Rosenberg, P., Horvath, G. \& Peterson, C. mdr-1 single nucleotide polymorphisms in ovarian cancer tissue: G2677T/A correlates with response to paclitaxel chemotherapy. Clin Cancer Res 12, 854-859 (2006).

6. Tian, C. et al. Common variants in ABCB1, ABCC2 and ABCG2 genes and clinical outcomes among women with advanced stage ovarian cancer treated with platinum and taxane-based chemotherapy: a Gynecologic Oncology Group study. Gynecol Oncol 124, 575-581 (2012).

7. Bergmann, T. K. et al. Impact of CYP2C $8 * 3$ on paclitaxel clearance: a population pharmacokinetic and pharmacogenomic study in 93 patients with ovarian cancer. Pharmacogenomics J 11, 113-120 (2011).

8. Johnatty, S. E. et al. ABCB1 (MDR 1) polymorphisms and progression-free survival among women with ovarian cancer following paclitaxel/carboplatin chemotherapy. Clin Cancer Res 14, 5594-5601 (2008).

9. Panczyk, M. et al. ABCB1 gene polymorphisms and haplotype analysis in colorectal cancer. Int J Colorectal Dis 24, 895-905 (2009).

10. Yang, J. J. et al. Genome-wide association study identifies germline polymorphisms associated with relapse of childhood acute lymphoblastic leukemia. Blood 120, 4197-4204 (2012).

11. Levy, P. et al. Influence of ABCB1 polymorphisms and docetaxel pharmacokinetics on pathological response to neoadjuvant chemotherapy in breast cancer patients. Breast Cancer Res Treat 139, 421-428 (2013).

12. Obata, H., Yahata, T., Quan, J., Sekine, M. \& Tanaka, K. Association between single nucleotide polymorphisms of drug resistance-associated genes and response to chemotherapy in advanced ovarian cancer. Anticancer Res 26, 2227-2232 (2006).

13. Drach, D. et al. Subpopulations of normal peripheral blood and bone marrow cells express a functional multidrug resistant phenotype. Blood 80, 2729-2734 (1992). 
14. Skipper, H. E., Schabel, F. M., Jr. \& Wilcox, W. S. Experimental Evaluation of Potential Anticancer Agents. Xiii. On the Criteria and Kinetics Associated with "Curability" of Experimental Leukemia. Cancer Chemother Rep 35, 1-111 (1964).

15. Hitzl, M. et al. The C3435T mutation in the human MDR1 gene is associated with altered efflux of the P-glycoprotein substrate rhodamine 123 from CD56+ natural killer cells. Pharmacogenetics 11, 293-298 (2001).

16. Wong, M. et al. Hepatic technetium Tc $99 \mathrm{~m}$-labeled sestamibi elimination rate and ABCB1 (MDR1) genotype as indicators of ABCB1 (P-glycoprotein) activity in patients with cancer. Clin Pharmacol Ther 77, 33-42 (2005).

17. Fojo, A. T. et al. Expression of a multidrug-resistance gene in human tumors and tissues. Proc Natl Acad Sci U S A 84, 265-269 (1987).

18. Park, J. G. et al. MDR1 gene expression: its effect on drug resistance to doxorubicin in human hepatocellular carcinoma cell lines. J Natl Cancer Inst 86, 700-705 (1994).

19. Efferth, T., Lohrke, H. \& Volm, M. Correlations between natural resistance to doxorubicin, proliferative activity, and expression of P-glycoprotein 170 in human kidney tumor cell lines. Urol Res 18, 309-312 (1990).

20. Guichard, S. M., Macpherson, J. S., Thurston, D. E. \& Jodrell, D. I. Influence of Pglycoprotein expression on in vitro cytotoxicity and in vivo antitumour activity of the novel pyrrolobenzodiazepine dimer SJG-136. Eur J Cancer 41, 1811-1818 (2005)

21. Johnatty, S. E. et al. ABCB1 (MDR1) polymorphisms and ovarian cancer progression and survival: a comprehensive analysis from the Ovarian Cancer Association Consortium and The Cancer Genome Atlas. Gynecol Oncol 131, 8-14 (2013).

22. Domcke, S., Sinha, R., Levine, D. A., Sander, C. \& Schultz, N. Evaluating cell lines as tumour models by comparison of genomic profiles. Nat Commun 4, 2126 (2013).

23. Slamon, D. J. et al. Studies of the HER-2/neu proto-oncogene in human breast and ovarian cancer. Science 244, 707-712 (1989).

24. Orr, G. A., Verdier-Pinard, P., McDaid, H. \& Horwitz, S. B. Mechanisms of Taxol resistance related to microtubules. Oncogene 22, 7280-7295 (2003).

25. Smoter, M. et al. The role of Tau protein in resistance to paclitaxel. Cancer Chemother Pharmacol 68, 553-557 (2011).

26. Ertel, A., Verghese, A., Byers, S. W., Ochs, M. \& Tozeren, A. Pathway-specific differences between tumor cell lines and normal and tumor tissue cells. Mol Cancer 5, 55 (2006).

27. Stein, W. D., Litman, T., Fojo, T. \& Bates, S. E. A Serial Analysis of Gene Expression (SAGE) database analysis of chemosensitivity: comparing solid tumors with cell lines and comparing solid tumors from different tissue origins. Cancer Research 64, 2805-2816 (2004).

28. Green, H. et al. Pharmacogenetic studies of Paclitaxel in the treatment of ovarian cancer. Basic Clin Pharmacol Toxicol 104, 130-137 (2009).

29. Yamaguchi, H. et al. Genetic variation in ABCB1 influences paclitaxe pharmacokinetics in Japanese patients with ovarian cancer. Int J Gynecol Cancer 16, 979-985 (2006).

30. Tothill, R. W. et al. Novel molecular subtypes of serous and endometrioid ovarian cancer linked to clinical outcome. Clin Cancer Res 14, 5198-5208 (2008).
31. Haber, M. et al. Association of high-level MRP1 expression with poor clinical outcome in a large prospective study of primary neuroblastoma. J Clin Oncol 24, 1546-1553 (2006).

\section{Acknowledgments}

This project was supported by the National Health and Medical Research Counci (NHMRC) of Australia (scholarship to B.G.), Cancer Institute New South Wales and Cancer Australia (552481). AdeF is funded by the University of Sydney Cancer Research Fund and the Cancer Institute NSW through the Sydney-West Translational Cancer Research Centre.

We gratefully acknowledge the cooperation of the institutions that supported the study. We also acknowledge the contribution of Australian Ovarian Cancer Study Group (full list can be found at http://www.aocstudy.org), all study nurses and research assistants and would like to thank all of the women who participated in AOCS. AOCS was supported by U.S. Army Medical Research and Materiel Command DAMD17-01-1-0729, NHMRC Enabling Grant 400413 and Project Grant 400281, the Cancer Council Tasmania and the Cancer Foundation of Western Australia.

\section{Author contributions}

B.G., R.B. and A.deF. wrote the main manuscript and were responsible for project design and conduct; B.G. conducted most of the experiments and prepared Figures 1-5, and Supplementary Figures 1 and 2; A.R., M.He., M.Ha. and M.N. contributed to data for Figures 1, 3, 5 and Supplementary Figure 2. J.B., X.Q.C., S.H., S.J. and G.C.-T. contributed to data for Figure 5 and Supplementary Figure 2; the Australian Ovarian Cancer Study, L.G. and D.B. provided study materials used in Figures 1-5, and Supplementary Figures 1 and 2; M.W., C.E. and P.H. contributed to data analysis and interpretation. All authors reviewed the manuscript.

\section{Additional information}

Supplementary information accompanies this paper at http://www.nature.com/ scientificreports

Competing financial interests: The authors declare no competing financial interests.

How to cite this article: Gao, B. et al. Paclitaxel sensitivity in relation to $A B C B 1$ expression, efflux and single nucleotide polymorphisms in ovarian cancer. Sci. Rep. 4, 4669; DOI:10.1038/srep04669 (2014)

This work is licensed under a Creative Commons Attribution-NonCommercialNoDerivs 3.0 Unported License. The images in this article are included in the article's Creative Commons license, unless indicated otherwise in the image credit; if the image is not included under the Creative Commons license, users will need to obtain permission from the license holder in order to reproduce the image. To view a copy of this license, visit http://creativecommons.org/licenses/by-nc-nd/3.0/ 
*Members of the Australian Ovarian Cancer Study Group:

David Bowtell ${ }^{1,2}$, Georgia Chenevix-Trench ${ }^{3}$, Anna deFazio ${ }^{4}$, Dorota Gertig ${ }^{5}$, Adèle Green ${ }^{3}$, Penelope Webb ${ }^{3}$, Jillian Hung ${ }^{4}$, Sue Moore ${ }^{3}$, Nadia Traficante', Sian Fereday', Karen Harrap ${ }^{3}$, Troy Sadkowsky ${ }^{3}$, Nirmala Pandeya ${ }^{3}$, Robin Stuart-Harris ${ }^{6}$, Fred Kirsten ${ }^{7}$, Josie Rutovitz ${ }^{8}$, Peter Clingan ${ }^{9}$, Amanda Glasgow ${ }^{9}$, Anthony Proietto ${ }^{10}$, Stephen Braye ${ }^{10}$, Greg Otton ${ }^{10}$, Jennifer Shannon ${ }^{11}$, Tony Bonaventura ${ }^{12}$, James Stewart ${ }^{12}$, Stephen Begbie ${ }^{13}$, Michael Friedlander ${ }^{14}$, David Bell ${ }^{15}$, Sally Baron-Hay ${ }^{15}$, Alan Ferrier ${ }^{15}$ (dec.), Greg Gard ${ }^{15}$, David Nevell ${ }^{15}$, Nick Pavlakis ${ }^{15}$, Sue Valmadre ${ }^{15}$, Barbara Young ${ }^{15}$, Catherine Camaris ${ }^{16}$, Roger Crouch ${ }^{16}$, Lyndal Edwards ${ }^{16}$, Neville Hacker ${ }^{16}$, Donald Marsden ${ }^{16}$, Greg Robertson ${ }^{16}$, Phillip Beale ${ }^{17}$, Jane Beith ${ }^{17}$, Jonothan Carter $^{17}$, Chris Dalrymple ${ }^{17}$, Anne Hamilton ${ }^{17}$, Roger Houghton ${ }^{17}$, Peter Russell ${ }^{17}$, Matthew Links ${ }^{18}$, John Grygiel ${ }^{19}$, Jane Hill ${ }^{20}$, Alison Brand ${ }^{21}$, Karen Byth ${ }^{21}$, Richard Jaworski ${ }^{21}$, Paul Harnett ${ }^{21}$, Raghwa Sharma ${ }^{21}$, Anita Achen ${ }^{21}$, Gerard Wain ${ }^{21}$, Bruce Ward 22 , David Papadimos ${ }^{22}$, Alex Crandon ${ }^{23}$, Margaret Cummings ${ }^{23}$, Ken Horwood ${ }^{23}$, Andreas Obermair ${ }^{23}$, Lew Perrin ${ }^{23}$, David Wyld ${ }^{23}$, Jim Nicklin ${ }^{23,24}$, Margaret Davy ${ }^{25}$, Martin K Oehler ${ }^{25}$, Chris Hall ${ }^{25}$, Tom Dodd ${ }^{25}$, Tabitha Healy ${ }^{25}$, Ken Pittman ${ }^{25}$, Doug Henderson ${ }^{26}$, John Miller ${ }^{27}$, John Pierdes ${ }^{27}$, Penny Blomfield ${ }^{28}$, David Challis ${ }^{28}$, Robert Mclntosh ${ }^{28}$, Andrew Parker ${ }^{28}$, Bob Brown ${ }^{29}$, Robert Rome ${ }^{29}$, David Allen ${ }^{30}$, Peter Grant ${ }^{30}$, Simon Hyde ${ }^{30}$, Rohan Laurie ${ }^{30}$, Melissa Robbie ${ }^{30}$, David Healy ${ }^{31}$, Tom Jobling $^{31}$, Tom Manolitsas ${ }^{31}$, Jane McNealage ${ }^{31}$, Peter Rogers ${ }^{31}$, Beatrice Susi ${ }^{31}$, Eric Sumithran ${ }^{31}$, lan Simpson ${ }^{31}$, Kelly Phillips ${ }^{1}$, Danny Rischin', Stephen Fox', Daryl Johnson', Paul Waring', Stephen Lade', Maurice Loughrey', Neil O'Callaghan', William Murray' ${ }^{1}$ Virginia Billson ${ }^{32}$, Jan Pyman ${ }^{32}$, Debra Neesham ${ }^{32}$, Michael Quinn ${ }^{32}$, Craig Underhill ${ }^{33}$, Richard Bell ${ }^{34}$, Leong-Fook Ng ${ }^{35}$, Robert Blum ${ }^{36}$, Vinod Ganju ${ }^{37}$, lan Hammond ${ }^{38}$, Yee Leung ${ }^{38}$, Anthony McCartney ${ }^{39}$ (dec.), Martin Buck ${ }^{40}$, Izak Haviv ${ }^{1}$, David Purdie $^{3}$, David Whiteman ${ }^{3}$, Nikolajs Zeps ${ }^{41,42}$, Mary-Rose Malt ${ }^{3}$, Anne Mellon ${ }^{10}$, Randall Robertson ${ }^{10}$, Trish Vanden Bergh ${ }^{16}$, Marian Jones ${ }^{16}$, Patricia Mackenzie ${ }^{16}$, Jane Maidens ${ }^{15}$, Kath Nattress ${ }^{17}$, Yoke-Eng Chiew ${ }^{4,21}$, Annie Stenlake ${ }^{21}$, Helen Sullivan ${ }^{21}$, Barbara Alexander ${ }^{3}$, Pat Ashover ${ }^{3}$, Sue Brown ${ }^{3}$, Tracy Corrish ${ }^{3}$, Lyn Green ${ }^{3}$, Leah Jackman ${ }^{3}$, Kaltin Ferguson ${ }^{3}$, Karen Martin ${ }^{3}$, Adam Martyn ${ }^{3}$, Barbara Ranieri ${ }^{3}$, Jo White $25,26,27$, Victoria Jayde $^{28}$, Leanne Bowes ${ }^{1}$, Pamela Mamers ${ }^{31}$, Laura Galletta', Debra Giles', Joy Hendley', Katherine Alsop', Trudy Schmidt ${ }^{41}$, Helen Shirley ${ }^{41}$, Colleen Ball ${ }^{41}$, Cherry Young ${ }^{41}$, Suzanna Viduka ${ }^{41}$, Hoa Tran ${ }^{41}$, Sanela Bilic ${ }^{41}$, Lydia Glavinas ${ }^{41}$, Julia Brooks ${ }^{42}$

'Peter MacCallum Cancer Centre, Melbourne, Australia, ${ }^{2}$ Sir Peter MacCallum Department of Oncology, Department of Pathology and Department of Biochemistry and Molecular Biology, University of Melbourne, Melbourne, Australia, ${ }^{3}$ QIMR Berghofer Medical Research Institute, Brisbane, Australia, ${ }^{4}$ Westmead Institute for Cancer Research, University of Sydney at Westmead Millennium Institute, Sydney, Australia, ${ }^{5}$ Victorian Cervical Cytology Registry, Melbourne, Australia, ${ }^{6} \mathrm{Canberra}$

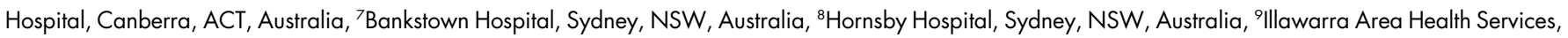
Wollongong, NSW, Australia, ${ }^{10}$ John Hunter Hospital, Newcastle, NSW, Australia, ${ }^{1}$ Nepean Hospital, Sydney, NSW, Australia, ${ }^{12}$ Newcastle Mater Misericordiae Hospital, Newcastle, NSW, Australia, ${ }^{13}$ Port Macquarie Base Hospital, Port Macquarie, NSW, Australia, ${ }^{14}$ Prince of Wales Hospital, Sydney, NSW, Australia, ${ }^{15}$ Royal North Shore Hospital, Sydney, NSW, Australia, ${ }^{16}$ Royal Hospital for Women, Sydney, NSW, Australia, ${ }^{17}$ Royal Prince Alfred Hospital, Sydney, NSW, Australia, ${ }^{18} \mathrm{St}$ George Hospital, Sydney, NSW, Australia, ${ }^{19} \mathrm{St}$ Vincent's Hospital, Sydney, NSW, Australia, ${ }^{20}$ Wagga Wagga Base Hospital, Wagga Wagga, NSW, Australia, ${ }^{21}$ Westmead Hospital, Sydney, NSW, Australia, ${ }^{22}$ Mater Misericordiae Hospitals, Brisbane, Queensland, Australia, ${ }^{23}$ Royal Women's Hospital, Brisbane, Queensland, Australia, ${ }^{24}$ Wesley Hospital, Brisbane, Queensland, Australia, ${ }^{25}$ Royal Adelaide Hospital and Burnside Memorial Hospital, Adelaide, SA, Australia, ${ }^{26}$ Flinders Medical Centre, Adelaide, SA, Australia, ${ }^{27}$ Queen Elizabeth Hospital, Adelaide, SA, Australia, ${ }^{28}$ Royal Hobart Hospital, Hobart, Tasmania, Australia, ${ }^{29}$ Freemasons Hospital, Melbourne, Victoria, Australia, ${ }^{30}$ Mercy Hospital for Women, Melbourne, Victoria, Australia, ${ }^{31}$ Monash Medical Centre, Melbourne, Victoria, Australia, ${ }^{32}$ Royal Women's Hospital, Melbourne, Victoria, Australia, ${ }^{33}$ Border Medical Oncology, Wodonga, Victoria, Australia, ${ }^{34}$ Andrew Love Cancer Centre, Geelong, Victoria, Australia, ${ }^{35}$ Ballarat Base Hospital, Ballarat, Victoria, Australia, ${ }^{36}$ Bendigo Health Care Group, Bendigo, Victoria, Australia, ${ }^{37}$ Peninsula Health, Frankston, Victoria, Australia, ${ }^{38}$ School for Women's and Infants' Health, University of Western Australia and the Western Australian Gynaecologic Cancer Service, Perth, WA, Australia, ${ }^{39}$ King Edward Memorial Hospital, Perth, WA, Australia, ${ }^{40}$ Mount Hospital, Perth, WA, Australia, ${ }^{41}$ WARTN, Western Australia Research Tissue Network, Perth, WA, Australia, ${ }^{42}$ St John of God Hospital, Perth, WA, Australia. 